\title{
Potencial utilitário da vegetação lenhosa em área de Caatinga no estado de Pernambuco, nordeste do Brasil
}

\author{
Utility of woody vegetation in a Caatinga area in the state of Pernambuco, northeastern Brazil \\ Cristiano Cardoso Gomes ${ }^{1}$ \\ Resumo
}

Este estudo teve como objetivo a análise da relação entre a população humana situada no Assentamento Olho d'Água do Félix, Caruaru-PE e as espécies florestais conhecidas e utilizadas, de forma a testar a hipótese de aparência ecológica. Foi realizado um inventário da vegetação arbórea através de parcelas amostrais de $400 \mathrm{~m}^{2}$ nos fragmentos florestais existentes no Assentamento. Os dados obtidos foram utilizados para calcular densidade, dominância, frequência e valor de importância. Compararam-se estes resultados com os dados etnobotânicos objetivando testar a hipótese de que a disponibilidade de um recurso (aparência) se relaciona com a sua importância para a população (determinada pelo valor de uso). Foram entrevistados 25 informantes-chaves que identificaram as espécies arbóreas nativas conhecidas e seus usos, estas foram apresentadas ao entrevistador através de turnê guiada. Vinte e sete espécies foram citadas e classificadas em nove categorias: alimentação humana, alimentação animal, combustível, construção, tecnologia, medicinal, veterinário, serviço ambiental e outros. As citações para usos não madeireiros da vegetação concentram o maior número de espécies, valor que foi influenciado pelas categorias alimentação animal e alimentação humana. Foi indicado o uso para as seguintes partes das plantas: entrecasca, flor, folha, fruto, látex, madeira, planta inteira, raize semente. O uso da madeira teve $74,8 \%$ das citações. As famílias com maior valor de uso foram Rhamnaceae e Anacardiaceae, já as famílias com maior número de espécies foram Fabaceae (9 spp.) e Euphorbiaceae (5 spp.). As espécies com maior valor de importância foram Croton blanchetianus Baill, Acacia farnesiana (L.) Willd. e Commiphora leptophloeos. As espécies de maior Valor de uso foram Mimosa tenuiflora Benth, Ziziphus joazeiro Mart. e Croton blanchetianus Baill. As categorias combustível, construção e medicinal foram as mais abundantes. Verificou-se que as espécies com menor número de partes da planta utilizadas tiveram menor valor de uso, e as que tiveram maior número de partes tiveram maior chance de terem maior número de usos. Percebeu-se que o índice combinado (VU x VI) tem correlação positiva com a densidade relativa, frequência relativa, valor de importância e dominância relativa. Foi significativa a correlação na ordenação das espécies entre o valor de uso e frequência relativa $(r=0,3260, p<0,01)$, e entre o valor de uso e a dominância relativa $(r=0,3987, \mathrm{p}<0,05)$. As espécies com menor número de partes utilizadas tiveram menor valor de uso, e as que tiveram maior número de partes tiveram maior chance de terem maior número de usos. A quantidade de usos, partes utilizadas e riqueza, demonstra o potencial utilitário da caatinga para as comunidades, e que esse conhecimento ecológico tradicional pode servir para prospecção de novos produtos e usos para produtos madeireiros e não madeireiros.

Palavras-chave: Aparência ecológica; Valor de uso; Etnobotânica; Caatinga

\begin{abstract}
This study had as its objective the analysis of the relationship between the human population located in the settlement of the Olho d'Água do Félix, Caruaru (Pernambuco state) and the forest species known and used, in order to test the hypothesis of ecological appearance. It was conducted an inventory of the arboreal vegetation through the sample plot of $400 \mathrm{~m}^{2}$ in the existing forest fragments in the settlement. The data obtained were used to calculate density, dominance, frequency, importance value. These results were compared with the Ethnobotanical data in order to test the hypothesis that the availability of a resource (appearance) is related to its importance for the population (determined by the value in use). Twenty-five key informants were interviewed and identified the native tree species and their uses. These ones were presented to the interviewer through a guided tour. Twenty-seven species have been cited and classified into nine categories: food, animal feed, fuel, construction, technology, medical, veterinary, environmental services and other uses. Quotations for non-timber uses of the vegetation concentrate the largest number of species, a value that was influenced by the categories animal feed and human food. It was indicated the use of the following plant parts: bast, flower, leaf, fruit, latex, wood, whole plant, root and seed. The use of wood had $74.8 \%$ of the quotes. Families with greater value in use were Rhamnaceae and Anacardiaceae, households with the largest number of species were Fabaceae ( 9 spp.) and Euphorbiaceae ( 5 spp.). The species with the highest value of importance were Croton blanchetianus Baill., Acacia farnesiana (1.) Willd. and Commiphora leptophloeos. The species of greatest value in use were Mimosa tenuiflora. Benth, Ziziphus Jacobus Mart Croton blanchetianus Baill. The categories fuel, construction and medical were the most abundant ones. It was verified that the species with less number of parts of the plant used had lower value of use, and those with greater number of parts were more likely to have a greater number of uses. It was noticed that the combined index (VU x VI) has a positive correlation with relative density, relative frequency, importance value and relative dominance. The correlation in the order of species between the value of use and relative frequency $(r=0.3260, p$ $<0.01)$ was significant, and between the value of use and the relative dominance $(r=0.3987, p<0,05)$. The species with the lowest number of parts used had lower value of use, and those with greater number of parts had a greater chance of having a greater number of uses. The amount of uses, used parts and wealth, demonstrates the caatinga utilitarian potential for the communities, and that this traditional ecological knowledge can be used to prospect new products and uses for wood and non-wood products.
\end{abstract}

Keywords: Ecological apparency hypothesis; Use value; Dry forest; Etnobotany

Engenheiro Florestal, MSc., Coordenador do Núcleo de Engenharia e Projetos, Sociedade Anônima de Águas e Esgoto do Crato, Rua Teodorico

Teles, 30, CEP 63100-160, Crato (CE), Brasil. sustentavel@gmail.com (ORCID: 0000-0003-3140-2179) 


\section{Introdução}

Os seres humanos percebem aquilo que de alguma forma está ligado às suas necessidades utilitárias, ecológicas e culturais (HUNN, 1982; MORRIS, 2000). A interação de humanos com os recursos naturais gera um conhecimento frequentemente denominado de "conhecimento ecológico tradicional", termo que faz referência, principalmente, à questão temporal da adaptação e transmissão desse conhecimento entre gerações (GADGIL; BERKES; FOLKE, 1993). Segundo Ellen (2006), as pessoas de qualquer tradição cultural interpretam, utilizam e, em geral, gerenciam seus conhecimentos sobre os domínios da experiência ambiental que englobam os organismos vivos e cujo estudo científico é delimitado pela zoologia, botânica e ecologia.

No semiárido brasileiro, as populações locais interagem com os recursos naturais e ampliam suas experiências no acesso à vegetação para coleta de produtos para sua subsistência e geração de renda, no entanto, nem sempre é sustentável. Por isso, compreender como ocorrem as relações entre a diversidade vegetal e a diversidade cultural é estratégico para ações de conservação. A etnobotânica é a ciência que buscar investigar essas relações (ALBUQUERQUE; HANAZAKI, 2009), e que encontra na diversidade biológica e cultural um interessante cenário para seu desenvolvimento (GALEANO, 2000; VANDEBROEK et al., 2004; GONZÁLEZ-INSUASTI; CABALLERO, 2006; KAWA; RODRIGUES; CILEMENT, 2011).

Diante disso algumas pesquisas têm demonstrado a importância em estudar as relações entre o conhecimento da população local sobre os recursos naturais e a disponibilidade de tais recursos (aparência ecológica), para compreender até onde a abundância de um dado recurso pode influenciar no seu uso. Na década de 90, Phillips e Gentry (1993a; 1993b) na tentativa de explicar a importância cultural das espécies vegetais, testaram a hipótese da aparência ecológica proposta inicialmente por Rhoades e Cates (1976) e por Feeny (1976) para explicar a relação entre herbívoros e plantas. Segundo os autores, espécies mais abundantes são fáceis de serem visualizadas e, portanto, de serem incorporadas aos sistemas de usos de povos locais (PHILLIPS; GENTRY, 1993a; 1993b).

Várias pesquisas tentam responder se plantas mais facilmente encontradas por seres humanos possuem maior chance de serem experimentadas e incluídas em um sistema cultural e, nesse sentido, se as plantas mais utilizadas por uma comunidade correspondem justamente àquelas mais "aparentes", ou seja, as mais fáceis de serem encontradas (ALBUQUERQUE et al., 2013).

Diante do exposto, o objetivo geral da pesquisa foi analisar a relação existente entre o valor de uso das espécies lenhosas nativas com os indicadores fitossociológicos e florísticos de um fragmento da caatinga localizada em um assentamento rural no município de Caruaru (Pernambuco) de forma a testar a hipótese de aparência ecológica. Os objetivos específicos foram (i) realizar um inventario florestal das espécies lenhosas da área de uso da comunidade; (ii) identificar as espécies lenhosas nativas arbóreas mais importantes para essa comunidade; (iii) analisar a diversidade de uso e a diversidade de plantas, e (v) relacionar o valor de uso com a disponibilidade das plantas no ambiente. Tais informações poderão colaborar para caracterizar padrões de uso dos recursos, cooperar no avanço de informações que possam contribuir para o melhor entendimento da aplicabilidade da aparência ecológica em florestas secas.

\section{Material e Métodos}

Área de estudo

A área selecionada para este estudo está localizada no Assentamento Fazenda Olho d'Água do Félix, Caruaru-PE, Nordeste do Brasil. O assentamento foi criado em 2001 pelo Instituto de Terras e Reforma Agrária do estado de Pernambuco através do Programa de Crédito Fundiário.

O município de Caruaru (Pernambuco) está situado na mesorregião Agreste e na Microrregião Vale do Ipojuca do Estado de Pernambuco, limitando-se ao Norte com Toritama, Vertentes, Frei Miguelino e Taquaritinga do Norte; ao Sul, com Altinho e Agrestina; a Leste, com Bezerros e Riacho das Almas; e a Oeste, com Brejo da 
Madre de Deus e São Caetano (COMPANHIA DE PESQUISA DE RECURSOS MINERAIS, 2005).

Segundo a classificação de Köppen, o clima da região é transicional entre o As (clima tropical com estação seca de verão) e o BSHw (clima das estepes quentes de baixa latitude e altitude). A precipitação média anual é de $550 \mathrm{~mm}$, com um máximo de $907 \mathrm{~mm}$ e um mínimo de $188 \mathrm{~mm}$. Em 2012, a precipitação no assentamento foi de apenas $323 \mathrm{~mm}$ (AGÊNCIA PERNAMBUCANA DE ÁGUAS E CLIMA, 2012). A temperatura varia entre $35^{\circ} \mathrm{C}$ no verão e $20^{\circ} \mathrm{C}$ no inverno. $\mathrm{O}$ relevo municipal possui superfícies suaves a onduladas, os solos ocorrentes na região são planossolos medianamente profundos, fortemente drenados, ácidos a moderadamente ácidos e de fertilidade natural média. Nos vales dos rios e riachos ocorrem os planossolos medianamente profundos, imperfeitamente drenados, textura médio argilosa, moderadamente ácidos com fertilidade natural alta e problemas de sais, encontrando-se ainda muitos afloramentos de rochas (CONSELHO DE DESENVOLVIMENTO DE PERNAMBUCO, 2002). A fitofisionomia estudada é a caatinga arbórea hipoxerófila, cujos componentes arbóreos de maior destaque são das famílias Fabaceae, Euphorbiaceae, Cactacea, Capparaceae e Rubiaceae. A vegetação arbórea apresenta elevada riqueza de Fabaceae e Euphorbiaceae, tal como encontrado por Alcoforado-Filho, Sampaio e Rodal (2003).

\section{Comunidade estudada}

A comunidade estudada está situada no Assentamento Olho d'Água do Félix. O assentamento possui 30 lotes e uma área de 703,24 hectares. Os lotes variam de 14 a 15 hectares. No assentamento, há quatro reservas legais, totalizando uma área de 141,069 hectares. A reserva legal faz fronteira com diferentes lotes dos assentados, alguns lotes ainda apresentam vegetação lenhosa, o que possibilita o uso e o contato direto com os recursos florestais. Apenas três lotes não fazem contato com a Área de Preservação Permanente que tem 36,45 ha. As reservas legais e cobertura florestal do assentamento encontram-se em diferentes graus de antropização e dão aos assentados diferentes formas de interação com os recursos florestais. As famílias assentadas são oriundas, principalmente, dos municípios de Caruaru e Toritama.

Dos 30 lotes existentes, apenas 29 possuem residentes. Cada lote apresenta uma família. As principais atividades agrícolas são o cultivo de milho e feijão, a manutenção de quintais produtivos com fruteiras, hortaliças e plantas medicinais, além de criação de pequenos animais, como galinhas, suínos, caprinos e bovinos. Eventualmente os assentados trabalham em atividades ligadas ao setor têxtil, seja no corte de tecidos ou na costura de roupas.

Dos 120 residentes de diferentes faixas etárias foram entrevistadas 45 pessoas, das quais 26 foram homens e 19 mulheres (23 adultos, 11 idosos e 11 jovens). As faixas etárias foram de acordo com a metodologia estabelecida pela pesquisa Nacional por Amostra de Domicílios do Instituto Brasileiro de Geografia e Estatística (IBGE, 2013) que considera jovens a faixa entre 15 a 29 anos de idade; como adultos a faixa de 29 a 59 anos de idade, e idosos acima de 60 anos.

Os adultos representam $51,11 \%$ dos amostrados. A idade dos entrevistados variou de 15 a 76 anos. Do grupo entrevistado, 64\% são naturais de Caruaru, 14,55\% de Toritama, e 5,45\% são oriundos de outros estados (AL, PB e SP), os demais 16\% são de municípios distantes de um raio de até $150 \mathrm{~km}$ de Caruaru (Ibirajuba, Tacaimbó, Timbaúba, Camocim de São Félix, Riacho das Almas, São Caetano, Santa Maria do Cambucá e Surubim). Dos entrevistados, 56,36\% moram no município há mais de 20 anos, e 70\% residem na comunidade há mais de dez anos.

Entre os entrevistados, 36,36\% indicaram não ter nenhum ano de escolaridade, e 64,54\% disseram ter até cinco anos de escolaridade. Muitos afirmaram saber apenas assinar seus nomes, considerando-se analfabetos.

Alguns dos entrevistados informaram terem exercido ou possuírem habilidade para as funções de cortador de tecido, costureiro, mecânico de máquinas de costura, servente de pedreiro, vendedor de chinelos, vendedor de confecções (sulanqueiro), vaqueiro, sapateiro e vigilante. Entre os entrevistados, $20 \%$ já migraram em algum momento da vida, São Paulo e Rio de Janeiro são os estados mais frequentes. Inventário etnobotânico 
Inicialmente, foi realizada uma reunião com os assentados para explicar os objetivos da pesquisa e estimular a participação das famílias no estudo. Para cada uma das pessoas foi lido o Termo de Consentimento Livre e Esclarecido, e a partir da aceitação dos mesmos em participar da pesquisa, foram convidados a assinar o termo, seguindo as exigências da Resolução n. 196 de 10/10/1996 do Conselho Nacional de Saúde.

Foram entrevistadas pessoas pertencentes a 28 famílias, visto que uma família não se dispôs em participar da pesquisa e a outra não estava no assentamento nos momentos em que foram realizadas as visitas. Um roteiro semiestruturado de entrevista foi utilizado para levantar as características socioeconômicas dos informantes, como idade, escolaridade, naturalidade, ocupação profissional, tempo de residência, outras habilidades além da agricultura e ocorrência de migração. Em seguida, aplicou-se a técnica de lista-livre a partir de uma pergunta geradora de dados que foi: "quais plantas você conhece?". Após o entrevistado informar não se lembrar de mais plantas, foi aplicada a técnica de uma nova leitura para revisão e possível enriquecimento da mesma (ALBUQUERQUE et al., 2008). A lista anteriormente mencionada pelo entrevistado foi lida pausadamente pelo entrevistador com o intuito do entrevistado lembrar-se de outras plantas. Para cada entrevistado, a lista foi lida até três vezes.

Essa etapa foi realizada entre janeiro de 2011 e abril de 2012, e todas informações fornecidas foram registradas em caderneta de campo e gravadas em gravador digital.

Durante a entrevista buscou-se evitar a influência direta de terceiros, isto para que as informações fossem pessoais, visto que, em muitas famílias mais de uma pessoa foi entrevistada.

No final das entrevistas foi realizada a técnica de turnê guiada, na qual os informantes foram solicitados a apresentar as plantas citadas na lista-livre (ALBUQUERQUE; LUCENA, 2010). Nesta oportunidade, além da coleta de material botânico, classificaram-se as plantas citadas quanto aos seus hábitos (erva, trepadeira, arbusto e árvore). Esta classificação também foi realizada durante o inventário florestal.

As plantas foram agrupadas quanto a sua origem, tendo-se considerado como plantas exóticas aquelas que não têm origem no Brasil, e como nativas aquelas originadas no Brasil. Este procedimento teve como objetivo classificar as espécies citadas e desta forma separar as de hábito arbóreo e arbustivo e as de ocorrência nativa, buscando aprofundar com parte dos mesmos os usos das arbóreas nativas. As informações auxiliaram a compreender quais espécies apresenta maior uso dos entrevistados e se estes têm correlação com a estrutura e a composição da vegetação.

A partir do desempenho dos entrevistados na primeira etapa, foram selecionados os que apresentaram maior número de citações de plantas nativas com hábito arbóreo e arbustivo, entre os diferentes sexos e nas faixas etárias de jovens, adultos e idosos. Foram selecionadas para o grupo de informantes-chave 25 pessoas, sendo 14 homens e 11 mulheres, dos quais 3 são jovens, 16 adultos e 6 idosos. A estes informanteschave foi apresentada a lista-livre anteriormente informada, e nesta oportunidade questionou-se sobre quais os usos da espécie e que parte é utilizada, considerando aqui apenas as espécies lenhosas nativas (arbóreas e arbustivas).

\section{Amostragem de vegetação}

Para obter dados sobre a estrutura e composição da vegetação foi realizado um levantamento fitossociológico entre junho de 2012 e fevereiro de 2013 através de parcelas alocadas nas áreas de reserva legal do assentamento. A metodologia utilizada para o inventário florestal, diâmetro de inclusão e medição das parcelas seguiu as orientações do Protocolo do Comitê Científico da Rede de Manejo Florestal da Caatinga (2005). Utilizou-se a parcela em formato quadrado com tamanho de 20 x $20 \mathrm{~m}$ (área de 400 $\mathrm{m}^{2}$ ), os indivíduos lenhosos vivos e mortos com DAP (Diâmetro a Altura do Peito) a partir de $2 \mathrm{~cm}$ foram amostrados e coletado, o sistema de amostragem utilizado foi aleatório, tendo-se implantado 15 parcelas, o que representou uma área amostral total de 0,60 hectares. As parcelas foram alocadas distantes das áreas de borda e em duas das reservas legais.

Os indivíduos foram inicialmente identificados pelo nome popular e com a colaboração de informantes do assentamento foram categorizados como vivos ou mortos. Foram feitas medidas de 
diâmetro na altura do solo (DNS) a 0,3 m e diâmetro a altura do peito (DAP) a 1,3 m de todos os indivíduos. As medidas de diâmetro foram feitas com suta dendrométrica, para diâmetros de até $20 \mathrm{~cm}$, para diâmetros superiores utilizou-se fita métrica, realizando-se a conversão de circunferência para diâmetro. Com o auxílio de uma vara retrátil foi estimada a altura e com uso de GPS as parcelas foram georreferenciadas.

No levantamento, as plantas lenhosas foram identificadas conforme seus hábitos utilizando-se os parâmetros propostos por Begon, Townsend e Harper (2006), e ajustados à vegetação local, segundo Costa e Araújo (2007).

Durante o levantamento, as espécies foram coletadas ou descritas morfologicamente, sua identificação ocorreu por morfologia comparada, usando bibliografia especializada e análise das exsicatas para comparação usando o material herborizado do Herbário Sérgio Tavares do Departamento de Ciência Florestal da UFRPE.

Todos os espécimes foram organizados utilizando-se o sistema de classificação APG III, os binômios das espécies foram verificados por meio do checklist do Centro Nordestino de Informações sobre Plantas (CENTRO NORDESTINO DE INFORMAÇÕES SOBRE PLANTAS, 2009) e as espécies foram sinonimizadas com base no sistema de dados W3 Trópicos (MISSOURI BOTANICAL GARDEN, 2004) e em revisões taxonômicas recentes, também foram conferidas pela lista de espécies da Flora do Brasil a sua distribuição geográfica.

As informações foram colhidas em fichas de campo, alimentados no software Excel e importada para o software Mata Nativa (FUNDAÇÃO DE CIÊNCIA E TECNOLOGIA, 2006).

Os dados de diâmetro na altura do solo (DNS), diâmetro na altura do peito, altura, nome da espécie e famílias foram anotados e processados com uso do software Mata Nativa (FUNDAÇÃO DE CIÊNCIA E TECNOLOGIA, 2006).

Os parâmetros fitossociológicos para estrutura horizontal calculados foram: abundância, densidade Relativa (DR), frequência relativa (FR), dominância relativa (DoR) e valor de importância (VI). Os cálculos dos parâmetros foram realizados seguindo a metodologia proposta por Mueller-Dombois e Ellenberg (1974). Os dados fitossociológicos de número de espécies foram utilizados para a elaboração da curva de coleta, curva coletora é uma abordagem desenvolvida por Gotelli e Colwell (2001).

\section{Análise de dados}

O valor de uso para cada espécie, família e categoria foi calculado e usou-se a fórmula: $\mathrm{VU}=$ $\sum \mathrm{Ui} / \mathrm{n}$ descrita por Rossato et al. (1999) e Silva e Albuquerque (2004), em que: Ui = número de usos mencionados por cada informante, $\mathrm{n}=$ número total de informantes. Os valores de uso das espécies por categoria utilitária também foram calculados segundo a fórmula adotada por Rossato et al. (1999), em que: $\mathrm{VUc}=\sum \mathrm{VU} / \mathrm{nc}$, sendo $\mathrm{VUc}=$ valor de uso de cada espécie na categoria, $\mathrm{Nc}=$ número de espécies na categoria.

Depois de calculados, os valores de uso foram divididos em classes, essas classes com um intervalo (amplitude) de 0,5 uso, o número total de classes estabelecido foram cinco. Foi feita uma adaptação à metodologia proposta por Albuquerque, Andrade e Silva (2005) em decorrência de não ter uso superior a 2,5 .

Os usos citados foram agrupados nas seguintes categorias: Alimentação Animal, Alimentação humana, Combustível, Construção, Medicinal, Outros Usos, Serviço Ambiental, Tecnologia e Veterinária.

O teste G de Williams (SOKAL; RHOLF, 1995) foi utilizado para comparar a riqueza de táxons entre as citações e inventário florestal. Os números de espécies e citações de uso por categoria utilitária foram comparados pelo teste de qui-quadrado $\left(\mathrm{X}^{2}\right)$ em nível de $5 \%$ de probabilidade. Diferenças nos valores de uso atribuídos às espécies pelos diferentes gêneros (homens e mulheres) e entre categorias de uso, foram comparadas pelo teste de Kruskal-Wallis (SOKAL; RHOLF 1995).

A relação entre o inventário etnobotânico e o inventário florestal (teste da aparência) foi avaliada por meio de correlação linear de Pearson (SOKAL; RHOLF,1995) através da combinação entre o valor de Importância (VI) com o valor de uso (VU), avaliando-se a existência e correlação na ordenação das famílias quando comparadas pelos índices tomados isoladamente (TORRE-CUADROS; ISBELE, 
2003).

Os dados registrados foram sistematizados em planilha eletrônica no Microsoft Excel e depois importados para o BioEstat 5.0 (AYRES et al., 2007) para a realização dos cálculos dos estimadores e índices. Buscou-se testar e analisar a relação entre o uso e a disponibilidade local.

\section{Resultados e discussão}

\section{Composição florística e dados fitossociológicos}

$\mathrm{Na}$ área de estudo foram inventariados 2.622 indivíduos em 15 parcelas, distribuídos em 29 táxons pertencentes a 23 gêneros e 12 famílias. As famílias mais representativas foram: Fabaceae (9 spp.) e Euphorbiaceae (4 spp.). Alcoforado-Filho, Sampaio e Rodal (2003) estudando a vegetação caducifólia espinhosa arbórea em Caruaru (PE) também encontraram elevada diversidade de Fabaceae e Euphorbiaceae.

As espécies com maior número de indivíduos amostrado foram Croton blanchetianus Baill, Acacia farnesiana (L.) Willd. Poincianella pyramidalis (Tul.) L. P. Queiroz, Mimosa tenuiflora (Willd.) Poir e Mimosa ophthalmocentra Benth. No estudo de Alcoforado-Filho, Sampaio e Rodal (2003) em Caruaru, também foi verificada uma elevada densidade de Poincianella pyramidalis, e Lucena et al. (2012a) estudando área de caatinga em Caruaru encontraram elevado número de indivíduos para Croton blanchetianus Baill. As espécies que tiveram menor número de indivíduos foram Lantana microphylla Mart e Lantana camara L.

O número de espécies diferentes variou de 2 a 15 por parcelas, o baixo número de espécies pode ser reflexo da antropização, a área tem indícios de sobrepastoreio e exploração seletiva, com o corte de várias espécies de interesse comercial. O número de indivíduos variou de 5 a 306. As espécies mais abundantes Croton blanchetianus e Acacia farnesiana são espécies típicas de ambientes antropizados. Croton blanchetianus e Commiphora leptophloeos tiveram maior valor de importância (VI). O Croton blanchetianus teve sua posição influenciada pela densidade, enquanto a Commiphora leptophloeos pela dominância.

As duas famílias com maior valor de importância foram Euphorbiaceae e Fabaceae decorrentes de sua densidade. A Burseraceae foi a terceira família com maior valor de importância (VI), apesar de ter uma baixa frequência e densidade, esse VI alto é justificado por sua dominância alta. É comum encontrar Commiphora leptophloeos com diâmetros grandes, essa espécie geralmente não é cortada pelos agricultores, pois existe localmente um senso de preservação para a mesma, por ser uma espécie que abriga abelhas nativas.

\section{Riqueza de uso e de espécies}

O levantamento etnobotânico identificou 27 táxons úteis pertencentes a 12 famílias e 23 gêneros. As famílias com maior riqueza foram Fabaceae (8 spp.) e Euphorbiaceae (4 spp.), e a que teve menor riqueza foi Apocynaceae, Burseraceae, Malvaceae, Rhamnaceae, Olacaceae, estas com apenas uma espécie.

A família Verbenaceae apresentou o menor valor de uso $(\mathrm{VU}=0,13)$ enquanto Rhamnaceae apresentou o maior valor de uso (VU =1,74). Lucena et al. (2012a) estudando uma comunidade rural em Caruaru - PE também encontraram na Verbenaceae o menor valor de uso entre as famílias. As espécies Mimosa tenuiflora, Ziziphus joazeiro, Poincianella pyramidalis, Croton blanchetianus e Myracrodruon urundeuva tiveram o maior valor de uso, entre estas o Croton blanchetianus teve maior valor de importância e as demais estão entre as dez de maior valor de uso. Das cinco espécies de maior valor de uso o Croton blanchetianus, Mimosa tenuiflora e Poincianella pyramidalis estão entre as cinco espécies com maior densidade relativa, já a Mimosa tenuiflora, Croton blanchetianus e Myracrodruon urundeuva estão entre as que apresentaram maior dominância relativa. Entre as espécies de maior valor de uso, o Croton blanchetianus também esteve entre as maiores dominâncias relativa, densidade relativa e valor de uso. 
Entre as dez espécies com maior valor de uso, oito têm valor de uso maior que 1,0 uso, e cinco têm também maior valor de importância. O valor de uso (VU) alto da Poincianella pyramidalis pode ter influência em seu alto valor de importância, o que a torna mais disponível e facilita seu acesso.

Entre as espécies com maior valor de uso, tem-se o Ziziphus joazeiro, cujo alto VU não é amparado por nenhum indicador fitossociológico, apesar de não ser frequente nem abundante é uma espécie importante, geralmente não é cortada, pois a madeira não é boa, mas é muito útil para alimentação de animais e o homem (Tabela, 1).

Tabela 1 - Espécies lenhosas, com DAP $>2 \mathrm{~cm}$, usadas no Assentamento Olho d'Água do Félix, município de Caruaru (Pernambuco, Nordeste do Brasil). Resultados dos parâmetros fitossociológicos e do valor de uso de cada espécie.

Table 1 - Woody species with DBH> $2 \mathrm{~cm}$, used in the settlement Olho d'Água do Félix, Caruaru (Pernambuco state, northeastern Brazil). Results of phytosociological parameters and the value in use of each species.

\begin{tabular}{|c|c|c|c|c|c|c|c|}
\hline $\begin{array}{c}\text { Família } \\
\text { Espécie (Nome Vernacular) }\end{array}$ & VU & DR & DoR & FR & VI & $\mathbf{A B}$ & VI $x$ VU \\
\hline Anacardiaceae & 1,04 & 2,21 & 4,18 & 8,34 & 14,728 & 0,218 & 15,48 \\
\hline Myracrodruon urundeuva Fr. Allem.(Aroeira) & 1,11 & 0,99 & 3,28 & 2,5 & 6,772 & 0,171 & 7,52 \\
\hline Schinopsis brasiliensis Engl. (Baraúna) & 1,00 & 0,65 & 0,64 & 4,17 & 5,456 & 0,033 & 5,46 \\
\hline Spondias tuberosa Arr. Cam. (Umbuzeiro) & 1,00 & 0,57 & 0,26 & 1,67 & 2,5 & 0,014 & 2,5 \\
\hline Apocynaceae & 0,52 & 1,83 & 0,67 & 5,00 & 7,50 & 0,04 & 3,90 \\
\hline Aspidosperma pyrifolium Mart. (Pereiro) & 0,52 & 1,83 & 0,67 & 5,00 & 7,5 & 0,035 & 3,9 \\
\hline Burseraceae & 0,93 & 0,46 & 31,09 & 2,50 & 34,05 & 1,62 & 31,67 \\
\hline $\begin{array}{l}\text { Commiphora leptophloeos (Mart.) J. B. Gillet } \\
\text { (Imburana-de-cheiro) }\end{array}$ & 0,93 & 0,46 & 31,09 & 2,5 & 34,049 & 1,622 & 31,67 \\
\hline Cactaceae & 0,49 & 0,69 & 3,28 & 5,00 & 8,968 & 0,171 & 4,57 \\
\hline Cereus jamacaru DC. (Mandacaru) & 0,56 & 0,61 & 2,00 & 3,33 & 5,946 & 0,104 & 3,33 \\
\hline Pilosocereus pachycladus F. Ritter (Facheiro) & 0,41 & 0,08 & 1,28 & 1,67 & 3,022 & 0,067 & 1,24 \\
\hline Capparaceae & 0,22 & 0,8 & 0,54 & 3,34 & 4,673 & 0,028 & 1,14 \\
\hline Capparis hastata Jacq. (Feijão-bravo) & 0,37 & 0,57 & 0,47 & 1,67 & 2,711 & 0,025 & 1,00 \\
\hline Capparis yco Mart (Icó) & 0,07 & 0,23 & 0,07 & 1,67 & 1,962 & 0,003 & 0,14 \\
\hline Combretaceae & 0,19 & 0,96 & 0,57 & 5,00 & 6,522 & 0,03 & 2,07 \\
\hline Combretum leprosum Mart. (Morfumbo) & & 0,08 & 0,02 & 0,83 & 0,931 & 0,001 & 0 \\
\hline Thiloa glaucocarpa (Mart.) Eichler. (Sipaúba) & 0,37 & 0,88 & 0,55 & 4,17 & 5,591 & 0,029 & 2,07 \\
\hline Euphorbiaceae & 0,55 & 57,21 & 30,37 & 20,84 & 108,417 & 1,584 & 119,81 \\
\hline Croton blanchetianus Baill. (Marmeleiro) & 1,19 & 55,38 & 28,4 & 12,5 & 96,274 & 1,481 & 114,57 \\
\hline Croton rhamnifolius Willd. (Quebra-faca) & 0,04 & 0,27 & 0,12 & 2,5 & 2,89 & 0,006 & 0,12 \\
\hline Jatropha mollissima (Pohl) Baill. (Pinhão) & 0,70 & 1,22 & 0,78 & 4,17 & 6,17 & 0,041 & 4,32 \\
\hline Manihot dichotoma Ule. (Maniçoba) & 0,26 & 0,34 & 1,07 & 1,67 & 3,083 & 0,056 & 0,8 \\
\hline Fabaceae & 0,70 & 34,56 & 28,53 & 43,33 & 106,401 & 1,489 & 101,41 \\
\hline Acacia farnesiana (L.) Willd. (Jurema-branca) & 0,44 & 13,5 & 6,18 & 9,17 & 28,848 & 0,322 & 12,69 \\
\hline $\begin{array}{l}\text { Anadenanthera colubrina var. cebil (Griseb.) } \\
\text { Altschul (Angico) }\end{array}$ & 1,00 & 0,61 & 2,57 & 0,83 & 4,009 & 0,134 & 4,01 \\
\hline Bauhinia cheilantha (Bong.) Steud. (Mororó) & 0,37 & 2,67 & 1,47 & 5,00 & 9,138 & 0,077 & 3,38 \\
\hline
\end{tabular}


Tabela 1: Conclusão...

Table1: Conclusion...

\begin{tabular}{|c|c|c|c|c|c|c|c|}
\hline $\begin{array}{c}\text { Família } \\
\text { Espécie (Nome Vernacular) }\end{array}$ & $\mathbf{V U}$ & DR & DoR & FR & VI & $\mathbf{A B}$ & VI $x$ VU \\
\hline $\begin{array}{l}\text { Libidibia ferrea (Mart. ex Tull) L.P.Queiroz (Pau- } \\
\text { ferro) }\end{array}$ & 0,70 & 0,23 & 0,15 & 2,5 & 2,874 & 0,008 & 2,01 \\
\hline $\begin{array}{l}\text { Mimosa ophthalmocentra Benth. (Jurema-de- } \\
\text { embira) }\end{array}$ & 0,30 & 4,2 & 2,64 & 8,33 & 15,168 & 0,138 & 4,55 \\
\hline Mimosa sp 1 (Jurema-carcará) & 0,07 & 0,19 & 0,09 & 1,67 & 1,947 & 0,005 & 0,14 \\
\hline Mimosa sp 2 (Coração-de-negro) & & 0,08 & 0,02 & 0,83 & 0,929 & 0,001 & 0 \\
\hline Mimosa tenuiflora (Willd.) Poir. (Jurema-preta) & 2,07 & 5,26 & 12,42 & 5,00 & 22,679 & 0,648 & 46,95 \\
\hline $\begin{array}{l}\text { Poincianella pyramidais (Tul). L. P. Queiroz } \\
\text { (Catingueira) }\end{array}$ & 1,33 & 7,82 & 2,99 & 10,00 & 20,809 & 0,156 & 27,68 \\
\hline Malvaceae & 0,22 & 0,11 & 0,14 & 0,83 & 1,09 & 0,01 & 0,24 \\
\hline $\begin{array}{l}\text { Pseudobombax simplicifolium A. Royns } \\
\text { (Imbiratanha) }\end{array}$ & 0,22 & 0,11 & 0,14 & 0,83 & 1,086 & 0,007 & 0,24 \\
\hline Olacaceae & 0,26 & 0,76 & 0,46 & 1,67 & 2,89 & 0,02 & 0,75 \\
\hline Ximenia americana L (Ameixa) & 0,26 & 0,76 & 0,46 & 1,67 & 2,894 & 0,024 & 0,75 \\
\hline Rhamnaceae & 1,74 & 0,34 & 0,15 & 2,50 & 2,99 & 0,01 & 5,21 \\
\hline Ziziphus joazeiro Mart. (Juazeiro) & 1,74 & 0,34 & 0,15 & 2,5 & 2,992 & 0,008 & 5,21 \\
\hline Verbenacee & 0,13 & 0,08 & 0,02 & 1,66 & 1,77 & 0,002 & 0,23 \\
\hline Lantana camara L. (Chumbinho) & 0,19 & 0,04 & 0,01 & 0,83 & 0,885 & 0,001 & 0,17 \\
\hline Lantana microphylla Mart. (Alecrim) & 0,07 & 0,04 & 0,01 & 0,83 & 0,885 & 0,001 & 0,06 \\
\hline
\end{tabular}

Em que: VU = Valor de Uso; DR = Densidade Relativa; DoR = Dominância relativa; FR = Frequência relativa; VI = Valor de Importância. $A B$ = área basal.

Os usos foram divididos em classes de valor de uso, com amplitude de 0,5 uso. A classe de uso que apresentou maior número de espécies foi a classe I $(0,0-0,5)$, seguida pela classe II e III (as categorias apresentam-se com tendência de um "J" invertido, ou seja, quanto maior o valor de uso menor o número de espécies. Observou-se uma tendência de menor riqueza de espécies com valores de uso alto, nesse sentido, este grupo tende a sofrer maior pressão extrativista. O estudo realizado por Lucena et al. (2012b), apresentou um gráfico que apresenta a mesma tendência de "J" invertido encontrado nesse estudo.

Observou-se que espécies com até dois usos tiveram baixo valor de uso, estando essas na primeira classe de uso $(<0,5)$. Já espécies com até três partes utilizadas tiveram VU de até 1,0 , enquanto para quatro e cinco partes utilizadas não tiveram padrão.

Foi atribuído uso para espécies de 12 famílias. Seis famílias tiveram valor de uso médio até 0,5 usos, e seis maiores que 0,5 usos. A família com maior valor de uso foi Rhamnaceae $(1,74)$, e a de menor valor foi Verbenaceae $(0,13)$.

As categorias que se destacaram quanto ao número de citações de uso foram: Construção (123 citações), Medicinal (118 citações), Combustível (79 citações), Alimentação Animal (68 citações) e Tecnologia (23 citações).

A Commiphora leptophloeos foi mencionada por 13 dos 24 informantes-chave, enquanto o Croton blanchetianus por 17 dos 24 informantes-chave. O Ziziphus joazeiro e a Mimosa tenuiflora tiveram cada citação de 17 informantes, a espécie que teve menor número de informantes foi Capparisyco e Mimosa sp 1 (juremacarcará) cada uma com um informante. Cada espécie foi em média citada por 8,88 informantes-chave.

Em estudo na comunidade de Malhada de Pedra em Caruaru (LUCENA et al., 2012a) também encontrou as categorias combustível, construção e medicinal como as mais abundantes, este estudo também as encontrou como sendo as mais importantes, sendo diferente apenas a ordem de classificação das mesmas. 
Foram registradas 467 citações de usos, sendo a média de 17,30 usos por espécie. As três categorias mais abundante representam $68,25 \%$ das citações.

Ao relacionar número de espécies com o valor de uso para citações, foi verificado não existir diferença significativa entre as categorias de uso $\left(X^{2}=20,43, p<0,0001\right)$, ou seja, há relação direta entre o número de espécies e a soma do valor de uso.

Ao verificar o número de espécies por categoria, percebe-se que as categorias de maior importância foram medicinal (21), construção (20) e alimentação animal (20), ficando combustível (18) na quarta posição. Das cinco espécies com maior valor de uso, quatro têm como um dos usos a construção. Lucena et al. (2012a) também encontraram as categorias medicinal e construção como as mais diversificadas. O resultado demonstra que a categoria construção tem potencial utilitário. Nascimento et al. (2008) ao estudarem o uso de espécies empregadas na construção de cercas em uma localidade rural de Caruaru verificaram o potencial utilitário do recurso florestal para a comunidade e constataram que o recurso florestal é muito útil.

A maioria das espécies tem de 2 a 5 usos A maior diversidade de espécies está na categoria com três usos e a menor está nas categorias de um, sete e oito usos. Apenas cinco espécies estão presentes em mais de seis categorias de uso.

Schinopsis brasiliensis foi mencionada para cinco categorias de uso (três categorias de uso madeireiro e duas de uso não madeireiro), apesar de seu potencial madeireiro, segundo informações dos assentados seu corte não é mais feito. A espécie é uma árvore protegida pela Portaria n. $83-\mathrm{N}$, de 26 de setembro de 1991 o que a torna imune ao corte, exceto sobre manejo florestal sustentável.

Tabela 2 - Categorias de uso e parte das plantas lenhosas, usadas no Assentamento Olho d'Água do Félix, município de Caruaru (Pernambuco, Nordeste do Brasil).

Table 2- Use categories and part of woody plants with $\mathrm{DBH}>2 \mathrm{~cm}$, used in Settlement Olho d'Água do Félix, Caruaru (Pernambuco state, northeastern Brazil).

\begin{tabular}{|c|c|c|c|c|c|c|c|}
\hline $\begin{array}{c}\text { Família } \\
\text { Espécie (Nome Vernacular) }\end{array}$ & VU & DR & DoR & FR & VI & $\mathbf{A B}$ & VI $x$ VU \\
\hline Anacardiaceae & 1,04 & 2,21 & 4,18 & 8,34 & 14,728 & 0,218 & 15,48 \\
\hline $\begin{array}{l}\text { Myracrodruon urundeuva Fr. Allem. } \\
\text { (Aroeira) }\end{array}$ & 1,11 & 0,99 & 3,28 & 2,5 & 6,772 & 0,171 & 7,52 \\
\hline Schinopsis brasiliensis Engl. (Baraúna) & 1 & 0,65 & 0,64 & 4,17 & 5,456 & 0,033 & 5,46 \\
\hline Spondias tuberosa Arr. Cam. (Umbuzeiro) & 1 & 0,57 & 0,26 & 1,67 & 2,5 & 0,014 & 2,5 \\
\hline Apocynaceae & 0,52 & 1,83 & 0,67 & 5 & 7,5 & 0,04 & 3,9 \\
\hline Aspidosperma pyrifolium Mart. (Pereiro) & 0,52 & 1,83 & 0,67 & 5 & 7,5 & 0,035 & 3,9 \\
\hline Burseraceae & 0,93 & 0,46 & 31,09 & 2,5 & 34,05 & 1,62 & 31,67 \\
\hline $\begin{array}{l}\text { Commiphora leptophloeos (Mart.) J. B. } \\
\text { Gillet (Imburana-de-cheiro) }\end{array}$ & 0,93 & 0,46 & 31,09 & 2,5 & 34,049 & 1,622 & 31,67 \\
\hline Cactaceae & 0,49 & 0,69 & 3,28 & 5 & 8,968 & 0,171 & 4,57 \\
\hline Cereus jamacaru DC. (Mandacaru) & 0,56 & 0,61 & 2 & 3,33 & 5,946 & 0,104 & 3,33 \\
\hline $\begin{array}{l}\text { Pilosocereus pachycladus F. Ritter } \\
\text { (Facheiro) }\end{array}$ & 0,41 & 0,08 & 1,28 & 1,67 & 3,022 & 0,067 & 1,24 \\
\hline Capparaceae & 0,22 & 0,8 & 0,54 & 3,34 & 4,673 & 0,028 & 1,14 \\
\hline Capparis hastata Jacq. (Feijão-bravo) & 0,37 & 0,57 & 0,47 & 1,67 & 2,711 & 0,025 & 1 \\
\hline Capparis yco Mart (Icó) & 0,07 & 0,23 & 0,07 & 1,67 & 1,962 & 0,003 & 0,14 \\
\hline Combretaceae & 0,19 & 0,96 & 0,57 & 5 & 6,522 & 0,03 & 2,07 \\
\hline Combretum leprosum Mart. (Morfumbo) & & 0,08 & 0,02 & 0,83 & 0,931 & 0,001 & 0 \\
\hline
\end{tabular}


Tabela 2-Continua...

Table 2 -Continued...

\begin{tabular}{|c|c|c|c|c|c|c|c|}
\hline $\begin{array}{c}\text { Família } \\
\text { Espécie (Nome Vernacular) }\end{array}$ & $\mathbf{V U}$ & DR & DoR & FR & VI & $\mathbf{A B}$ & VI $x$ VU \\
\hline $\begin{array}{l}\text { Thiloa glaucocarpa (Mart.) Eichler. } \\
\text { (Sipaúba) }\end{array}$ & 0,37 & 0,88 & 0,55 & 4,17 & 5,591 & 0,029 & 2,07 \\
\hline Euphorbiaceae & 0,55 & 57,21 & 30,37 & 20,84 & 108,417 & 1,584 & 119,81 \\
\hline Croton blanchetianus Baill. (Marmeleiro) & 1,19 & 55,38 & 28,4 & 12,5 & 96,274 & 1,481 & 114,57 \\
\hline Croton rhamnifolius Willd. (Quebra-faca) & 0,04 & 0,27 & 0,12 & 2,5 & 2,89 & 0,006 & 0,12 \\
\hline Jatropha mollissima (Pohl) Baill. (Pinhão) & 0,7 & 1,22 & 0,78 & 4,17 & 6,17 & 0,041 & 4,32 \\
\hline Manihot dichotoma Ule. (Maniçoba) & 0,26 & 0,34 & 1,07 & 1,67 & 3,083 & 0,056 & 0,8 \\
\hline Fabaceae & 0,7 & 34,56 & 28,53 & 43,33 & 106,401 & 1,489 & 101,41 \\
\hline $\begin{array}{l}\text { Acacia farnesiana (L.) Willd. } \\
\text { (Jurema-branca) }\end{array}$ & 0,44 & 13,5 & 6,18 & 9,17 & 28,848 & 0,322 & 12,69 \\
\hline $\begin{array}{l}\text { Anadenanthera colubrina var. cebil } \\
\text { (Griseb.) Altschul (Angico) }\end{array}$ & 1 & 0,61 & 2,57 & 0,83 & 4,009 & 0,134 & 4,01 \\
\hline $\begin{array}{l}\text { Bauhinia cheilantha (Bong.) Steud. } \\
\text { (Mororó) }\end{array}$ & 0,37 & 2,67 & 1,47 & 5 & 9,138 & 0,077 & 3,38 \\
\hline $\begin{array}{l}\text { Libidibia ferrea (Mart. ex Tull) L.P.Queiroz } \\
\text { (Pau-ferro) }\end{array}$ & 0,7 & 0,23 & 0,15 & 2,5 & 2,874 & 0,008 & 2,01 \\
\hline $\begin{array}{l}\text { Mimosa ophthalmocentra Benth. } \\
\text { (Jurema-de-embira) }\end{array}$ & 0,3 & 4,2 & 2,64 & 8,33 & 15,168 & 0,138 & 4,55 \\
\hline Mimosa sp 1 (Jurema-carcará) & 0,07 & 0,19 & 0,09 & 1,67 & 1,947 & 0,005 & 0,14 \\
\hline Mimosa sp 2 (Coração-de-negro) & & 0,08 & 0,02 & 0,83 & 0,929 & 0,001 & 0 \\
\hline $\begin{array}{l}\text { Mimosa tenuiflora (Willd.) Poir. } \\
\text { (Jurema-preta) }\end{array}$ & 2,07 & 5,26 & 12,42 & 5 & 22,679 & 0,648 & 46,95 \\
\hline $\begin{array}{l}\text { Poincianella pyramidais (Tul). L. P. } \\
\text { Queiroz (Catingueira) }\end{array}$ & 1,33 & 7,82 & 2,99 & 10 & 20,809 & 0,156 & 27,68 \\
\hline Malvaceae & 0,22 & 0,11 & 0,14 & 0,83 & 1,09 & 0,01 & 0,24 \\
\hline $\begin{array}{l}\text { Pseudobombax simplicifolium A. Royns } \\
\text { (Imbiratanha) }\end{array}$ & 0,22 & 0,11 & 0,14 & 0,83 & 1,086 & 0,007 & 0,24 \\
\hline Olacaceae & 0,26 & 0,76 & 0,46 & 1,67 & 2,89 & 0,02 & 0,75 \\
\hline Ximenia americana L (Ameixa) & 0,26 & 0,76 & 0,46 & 1,67 & 2,894 & 0,024 & 0,75 \\
\hline Rhamnaceae & 1,74 & 0,34 & 0,15 & 2,5 & 2,99 & 0,01 & 5,21 \\
\hline Ziziphus joazeiro Mart. (Juazeiro) & 1,74 & 0,34 & 0,15 & 2,5 & 2,992 & 0,008 & 5,21 \\
\hline Verbenacee & 0,13 & 0,08 & 0,02 & 1,66 & 1,77 & 0,002 & 0,23 \\
\hline Lantana camara L. (Chumbinho) & 0,19 & 0,04 & 0,01 & 0,83 & 0,885 & 0,001 & 0,17 \\
\hline Lantana microphylla Mart. (Alecrim) & 0,07 & 0,04 & 0,01 & 0,83 & 0,885 & 0,001 & 0,06 \\
\hline
\end{tabular}

As espécies mais mencionadas foram Mimosa tenuiflora (56 citações), Ziziphus joazeiro (47 citações) e Poincianella pyramidalis (36 citações). Quanto ao produto do valor de VI x VU, a Croton blanchetianus, Mimosa tenuiflora, Commiphora leptophloeos e Poincianella pyramidalis estão entre os 
maiores índices da combinação de VI X VU.

\section{Aparência ecológica}

Das 29 espécies inventariadas, 27 tiveram uso informado pertencendo a 12 famílias e 23 gêneros. Existiu uma forte relação entre a riqueza total e riqueza de espécies úteis por família botânica e gênero, pois a comunidade citou uso para $93,10 \%$ das espécies presentes no inventário florestal.

A espécie que teve o maior valor de uso foi Mimosa tenuiflora (Willd.) Poir. (VU =2,07) e que apresentou menor valor foi Croton rhamnifolius $(\mathrm{VU}=0,07)$.

As duas espécies com maior valor de uso apresentaram pelos menos cinco partes utilizadas da planta, e foram categorizadas em no mínimo seis categorias. O que pode indicar que quanto mais partes utilizadas maior o uso e provável pressão sobre a espécie.

Quatro das cinco espécies com maior valor da combinação do valor de uso com o valor de importância (VU x VI) apresentaram maior dominância relativa, e três das cinco com maior frequência apresentaram também maiores valores da combinação do valor de uso com valor de importância.

O índice da combinação de VU x VI variou de 0,06 a 114,57, buscando facilitar a análise dos dados obtidos, os índices foram categorizados em intervalos de classes, com amplitude de 5 , tendo estabelecido cinco classes $(0,01$ a 5,0; 5,01 a 10,0;10,01 a 15,0; 15,01 a 20,00; e maior que 20). A classe I obteve a maior riqueza (20 espécies), a classe IV não teve nenhuma espécie, enquanto a classe III teve apenas uma espécie. A Classe V teve quatro espécies (Croton blanchetianus, Mimosa tenuiflora, Commiphora leptophloeos e Poincianella pyramidalis). Entre as cinco espécies com maior índice da combinação, três estão entre as maiores frequências e quatro têm os maiores índices de dominância relativa.

Não foi evidenciado para espécies correlação entre o valor de uso com o valor de importância $(\mathrm{r}=$ $0,3645, \mathrm{p}>0,05)$, e nem entre o valor de uso e densidade relativa $(\mathrm{r}=0,2629, \mathrm{p}>0,1)$. Não foi evidenciada para as famílias a correlação entre o valor de uso com o valor de importância $(r=0,1144, p>0,05)$.

Encontrou-se significativa correlação na ordenação das espécies entre o valor de uso e frequência relativa $(r=0,3260, p<0,01)$, e entre o valor de uso e a dominância relativa $(r=0,3987, p<0,05)$.

A ordenação das espécies entre o valor de uso e índice combinado (VU x VI) apresentou uma expressiva e significativa correlação $(r=0,5167, \mathrm{p}<0,01)$.

Foi encontrada forte correlação positiva para ordenação das espécies entre o índice combinado (VU x VI) e a densidade relativa $(\mathrm{r}=0,9106, \mathrm{p}<0,0001)$, índice combinado (VU x VI) e a frequência relativa $(\mathrm{r}=0,6840, \mathrm{p}<0,0001)$, o índice combinado (VU x VI) e a dominância relativa $(\mathrm{r}=0,8210, \mathrm{p}<$ 0,0001), o índice combinado (VU x VI) e o valor de importância $(r=0,9591, p<0,001)$.

Verifica-se que o índice combinado (VU x VI) tem correlação positiva com a densidade relativa, frequência relativa, valor de importância e dominância relativa. Ou seja, o índice combinado (VU X VI) é influenciado pelas variáveis independentes de densidade relativa, frequência relativa, valor de importância e dominância relativa. Foi encontrada uma forte e positiva correlação para ordenação das famílias entre o índice combinado (VU x VI) e a densidade relativa $(\mathrm{r}=0,9628, \mathrm{p}<0,0001)$.

Foi evidenciada uma associação moderada e não significativa entre frequência relativa e valor de uso $(r=0,2918 ; p>0,10)$ ao ser testada através da correlação de Pearson.

Ao juntar as categorias que usam madeira, identifica-se que $48.18 \%$ das citações têm fins madeireiros enquanto $51,82 \%$ das espécies tiveram uso não madeireiro. Das 27 espécies, 26 espécies tiveram uso madeireiro e 25 para não madeireiros, apenas três espécies tiveram uso exclusivamente não madeireiro e duas de uso exclusivamente madeireiro. Os dados encontrados divergem dos encontrados por Lucena et al. (2012a), pois no estudo dos autores, o uso madeireiro foi mais importante que o não madeireiro.

Apesar de os usos não madeireiros serem mais diversos que os usos madeireiros, o qual foi, sobretudo, influenciado pela contribuição da alimentação animal e em parte a alimentação humana, a diferença não é significativa $\left(\mathrm{x}^{2}=0,144, \mathrm{p}<0,001\right)$.

A contribuição do valor de uso médio das espécies por categoria variou de 0,04 a 0,26 . A categoria que teve a maior média de uso foi Outros usos, e a que apresentou menor valor de uso foi a categoria 
Veterinária. O teste de Kruskal-Wallis mostrou não serem as mesmas significativas. Provavelmente, esse fato se deve à presença das espécies com maior valor de uso em todas as categorias, o que pode homogeneizar os dados $(\mathrm{H}=5.2819, \mathrm{p}>0,05)$.

Na Colômbia Marin-Corba, Cárdenas-López e Suárez-Suárez (2005) em estudo com colonos evidenciaram que a categoria combustível apareceu com maior importância, para os autores tal resultado explica-se pelo fato que quase toda planta com caule lenhoso pode ser usada com propósito energético. Já Lucena et al. (2012a) verificaram que os usos mais importantes estão relacionados com a extração de madeira para fins energéticos e de construção.

As relações demonstram que a estrutura florestal pode estar influenciando o uso do recurso pelas populações locais. O resultado corrobora a pesquisa de Maldonado et al. (2013) que estudando comunidades rurais mexicanas em área de floresta seca, mostraram que as espécies mais utilizadas são as de maior importância ecológica.

\section{Conclusões}

A capacidade elevada de listar espécies e designar os seus usos, demonstra que assentados possuem um expressivo conhecimento sobre a vegetação, haja vista ter descrito uso para praticamente todas as espécies, além da capacidade de aproveitar as múltiplas partes das plantas lenhosas.

Apesar da grande colaboração da floresta para fitocombustíveis e madeira para construção, os assentados também usam a floresta para outros fins.

Foi evidenciada a associação entre a importância relativa de um recurso e a sua disponibilidade, sendo perceptíveis as seguintes implicações: 1) Das espécies disponíveis no ambiente $93,10 \%$ têm uso pela comunidade, demonstrando assim o grande potencial utilitário do fragmento; 2) Verifica-se que o índice combinado (VU x VI) tem correlação positiva com a densidade relativa, frequência relativa, valor de importância e dominância relativa. Ou seja, o índice combinado (VU X VI) é influenciado pelas variáveis independentes de densidade relativa, frequência relativa, valor de importância e dominância relativa; 3) A ordenação das espécies entre o valor de uso e índice combinado (VU x VI) apresentou uma expressiva e significativa correlação $(\mathrm{r}=0,5167, \mathrm{p}<0,01)$. 4) As espécies com menor número de partes utilizadas tiveram menor valor de uso, e as que tiveram maior número de partes tiveram maior chance de terem maior número de usos; 5) As espécies com menor número de indivíduos foram as que tiveram menor valor de uso.

Os resultados encontrados demonstram que há influência da vegetação sobre uso e conhecimento de algumas plantas, explicado pela correlação entre valor de uso e frequência, e valor de uso e dominância.

A quantidade de usos, partes utilizadas e riqueza demonstra o potencial utilitário da caatinga para as comunidades, e que esse conhecimento ecológico tradicional pode servir para prospecção de novos produtos e novos usos para produtos madeireiros e não madeireiros

\section{Agradecimento}

Ao Programa de Pós-graduação em Ciências Florestais da UFRPE e Assentados do Assentamento Olho d'Água do Félix (Caruaru-PE)

\section{Referências}

ALBUQUERQUE, U. P. et al. The current status of ethnobiological research in Latin America: gaps and perspectives. Journal of Ethnobiology and Ethnomedicice, London, v. 9, n. 72, 2013.

ALBUQUERQUE, U. P. et al. Métodos e técnicas na pesquisa etnobotânica. Recife: Comunigraf, 2008. $324 \mathrm{p}$.

ALBUQUERQUE, U. P.; ANDRADE, L. H. C.; SILVA, A. C. Use of plant resources in a seasonal dry forest (Northeastern Brazil). Acta Botanica Brasílica, Porto Alegre, v. 19, p. 27-38, 2005. 
ALBUQUERQUE, U. P.; HANAZAKI, N. Five problems in current ethnobotanical research - and some suggestions for strengthening them. Humam Ecology, New York, v. 37, p. 653-661, 2009.

ALBUQUERQUE, U. P.; LUCENA, R. F. P. Seleção e escolha dos informantes. In: ALBUQUERQUE, U. P.; LUCENA, R. F. P. (Org.). Métodos e técnicas na pesquisa etnobotânica. Recife: Comunigraf, 2010. p. 19-35.

ALCOFORADO-FILHO, F. G.; SAMPAIO, E. V. S. B.; RODAL, M. J. N. Florística e fitossociologia de um remanescente de vegetação caducifólia espinhosa arbórea em Caruaru, Pernambuco. Acta Botânica Brasílica, Porto Alegre, v. 17, n. 2, p. 287-303, 2003.

AGÊNCIA PERNAMBUCANA DE ÁGUAS E CLIMA. Bacias Hidrográficas. 2011. Disponível em: http://www.apac.pe.gov.br. Acesso em: 3 set. 2011.

AYRES, M. et al. BioEstat 5.0: aplicações estatísticas nas áreas das ciências bio-médicas. Belem: Ong Mamiraua, 2007.

BEGON, M.; TOWNSEND, C. R.; HARPER, J. L. Ecology: from individuals to ecosystems. 4th ed. Oxford: Blackwell, 2006.

CENTRO NORDESTINO DE INFORMAÇÕES SOBRE PLANTAS. Centro nordestino de informações sobre plantas. 2009. Disponível em: http://www.cnip.org.br. Acesso em: 11 nov. 2012.

COMITÊ TÉCNICO CIENTÍFICO DA REDE DE MANEJO FLORESTAL DA CAATINGA. Rede de manejo florestal da Caatinga: protocolo de medições de parcelas permanentes. Recife: Comitê Técnico Científico; Associação Plantas do Nordeste, 2005.

COMPANHIA DE PESQUISA DE RECURSOS MINERAIS. Projeto cadastro de fontes de abastecimento por água subterrânea. Diagnóstico do município de Caruaru, estado de Pernambuco. Recife: CPRM; PRODEEM, 2005. 33 p. Disponível em: http://www.cprm.gov.br acesso em 14 de maio de 2010. Acesso em: 1 set. 2011.

CONSELHO DE DESENVOLVIMENTO DE PERNAMBUCO. Base de dados do Estado: Climatologia: descrição dos tipos. Recife: Governo do Estado de Pernambuco; Instituto de planejamento de Pernambuco, 2000 .

COSTA, I. R.; ARAÚJO, F. S. Organização comunitária de um encrave de cerrado sensu stricto no Bioma Caatinga, Chapada do Araripe, Barbalha, Ceará. Acta Botânica Brasilica, Porto Alegre, v. 21, n. 2, p. 281-291, 2007.

ELLEN, R. F. The cultural relations of classification: an analysis of Nuaulu animal categories from central Seram Cambridge. Cambridge: University Pres, 2006. 344 p.

FEENY, P. Plant apparency and chemical defense. In: WALLACE, J. W.; NANSEL, R. L. (Ed.). Biological interactions between plants and insects. Recent Advances in Phytochemistry, New York, v. 10, p. 1-40, 1976.

FUNDAÇÃO DE CIÊNCIA E TECNOLOGIA (RS). Mata Nativa. 2: manual do usuário. Viçosa: CIENTEC, 2006.

GADGIL, M.; BERKES, F.; FOLKE, C. Indigenous knowledge for biodiversity conservation. Ambio, Washington, v. 22, n. 2/3, p. 151-156, 1993.

GALEANO, G. Forest use at the pacific coast of chocos Colombia: a quantitative approach. Economic Botany, Bronx, v. 54, n. 3, p. 358-376, 2000.

GONZÁLEZ-INSUASTI, M. S.; CABALLERO, J. Managing plant resources: how intensive can it be? 
Human Ecology, New York, v. 35, n. 3, p. 303-314, 2006.

GOTELLI, N.; COLWELL, R. K. Quantifying biodiverty: procedures and pitfalls in the measurement and comparison of species richness. Ecology Letters, Hoboken, v. 4, p. 379-391, 2001.

HUNN, E. The utilitarian factor in folk biological classification. American Anthropologist, Arlington, v. 84, n. 4, p. 830-847, 1982.

KAWA, N. C.; RODRIGUES, D.; CILEMENT, C. R. Useful species richness, proportion of exotic species, and market orientation on Amazonian dark earths and oxisols. Economic Botany, Bronx, v. 65, n. 2, p. 169-177, 2011.

IBGE. Pesquisa nacional por amostra de domicílios: síntese de indicadores 2013, Coordenação de Trabalho e Rendimento. 1. ed. Rio de Janeiro: IBGE, 2013.

LUCENA, R. F. P. et al. The ecological apparency hypothesis and the importance of useful plants in rural communities from Northeastern Brazil: an assessment based on use value. Journal of Environmental Management, Amsterdam, v. 96, p. 106-115, 2012a.

LUCENA, R. F. P. et al. O uso de espécies vegetais no vale do piancó pode ser explicado por sua disponibilidade local? Revista de Biologia e Farmácia, Campina Grande, nesp, 2012b.

MARÍN-CORBA, C.; CÁRDENAS-LÓPEZ, D.; SUÁREZ-SUÁREZ, S. Utilidad del valor de uso en etnobotánica. Caldasia, Bogotá, v. 27, n. 1, p. 89-101, 2005.

MISSOURI BOTANICAL GARDEN. W3 Trópicos, VAST - Vascular Trópicos. 2011. Disponível em: http://www.tropicos.org. Acesso em: 1 jun. 2011.

MORRIS, B. The pragmatics of folk classification. In: PAUL, E. M. (Ed.). Ethnobotany. Norman: University of Oklahoma Press, 2000. p. 69-87.

MUELLER-DOMBOIS, D.; ELLENBERG, H. Aims and methods of vegetation ecology. New York: John Wiley \& Sons, 1974. 547 p.

NASCIMENTO, V. T. et al. Rural fences in agricultural landscapes and their conservation role in an area of caatinga (dryland vegetation) in Northeast Brazil. Environment, Development and Sustainability, Berlin, v. 11, n. 5, p. 1005-1029, 2008.

PHILLIPS, O.; GENTRY, A. H. The useful plants of Tambopata, Peru: statistical hypotheses tests with a new quantitative technique. Economic Botany, Bronw, v. 47, n. 1, p. 15-32, 1993a.

PHILLIPS, O.; GENTRY, A. H. The useful plants of Tambopata, Peru: II. Additional hypothesis testing in quantitative etnobotany. Economic botany, Bronw, v. 47, n. 1, p. 33-43, 1993 b.

RHOADES, D. F.; CATES, R. G. Toward a general theory of plant antiherbivore chemistry. In: WALLAC, J. W.; NANSEL (Ed.). Biological 47 interactions between plants and insects: recent advances in phytochemistry. New York: Plenum Press, 1976. v. 10. p. 169-213.

ROSSATO, S.C. et al. Ethnobotany caiçaras of the atlantic forest coast (Brazil). Economic Botany, Bronw, NY, v. 53, 1999.

SILVA, V.A.; ALBUQUERQUE, U.P. Técnicas para análise de dados etnobotânicos. In: ALBUQUERQUE, U.P.; LUCENA, R.F.P. (Org.) Métodos e técnicas na pesquisa etnobotânica. p.63-88, 2004.

SOKAL R. R.; RHOLF F. G. Biometry. New York: Freeman and Company, 1995.

TORRE-CUADROS, M. A.; ISBELE, G. A. Traditional ecological knowledge and use of vegetaion in southeastern Mexico: a case study from Solferino, Quintana Roo. Biodiversity and Conservation, 
London, v. 12, p. 2455-2476, 2003.

VANDEBROEK, I. et al. Use of medicinal plants and pharmaceuticals by indigenous communities in the Bolivian Andes and Amazon. Bulletin World Health Organization, Geneva, v. 82, n. 4, p. 243-250, 2004.

Ci. Fl., Santa Maria, v. 29, n. 1, p. 307-321, jan./mar., 2019 